\section{Preemergent Herbicide Safety in Container-grown Ornamental Grasses}

\author{
Joseph C. Neal ${ }^{1}$ \\ Department of Floriculture and Ornamental Horticulture, Cornell \\ University, Ithaca, NY 14853
}

\author{
Andrew F. Senesac ${ }^{2}$ \\ Cornell University, Long Island Horticultural Research Laboratory, \\ Riverhead, NY 11901
}

Additional index words. benefin, isoxaben, metolachlor, MON 15151, napropamide, oryzalin, oxadiazon, oxyfluorfen, pendimethalin, prodiamine, trifluralin, weed control, beach grass, blue fescue, fountain grass, pampas grass, ribbon grass, Ammophila breviligulata, Cortaderia selloana, Deschampsia caespitosa, Festuca ovina glauca, Pennisetum setaceum, Phalaris arundinacea picta

Abstract. Preemergent herbicide phytotoxicity was evaluated for six species of container-grown ornamental grasses: beach grass (Ammophila breviligulata Fern.), pampas grass [Cortaderia selloana (Schult. \& Schult. f.) Asch. \& Graebn.], tufted hair grass [Deschampsia caespitosa (L.) Beauvois.], blue fescue [Festuca ovina cv. glauca (Lam.) W.D.J. Koch], fountain grass [Pennisetum setaceum (Forssk.) Chiov.], and ribbon grass (Phalaris arundinacea cv. picta L.). Herbicides included isoxaben, metolachlor, MON 15151, napropamide, oryzalin, oxadiazon, pendimethalin, prodiamine, and trifluralin; the granular combination products of benefin plus trifluralin; and oxyfluorfen plus pendimethalin. Metolachlor, granular or spray, and oryzalin severely injured all species tested, except beachgrass, which was not injured by metolachlor granule. Napropamide injured pampas grass, fountain, grass, blue fescue, and tufted hair grass, but was safe on ribbon grass and beach grass. Pendimethalin, prodiamine, trifluralin; MON 15151, isoxaben, oxyfluorfen plus pendimethalin, and benefin plus trifluralin were safe on all six species. Chemical names used: N-butyl-N-ethyl-2,6-dinitro-4-(trifluoromethyl)benzenamine(benefin); $\mathrm{N}$-[3-(1-ethyl-1-methylpropyl)5-isoxazolyl]-2,6dimethoxybenzamide(isoxaben);2-chloro- $\mathrm{N}$-(2-ethyl-6-methylphenyll-N-(2-methoxy-1methylethyl)acetamide (metolachlor); S,S-dimethyl 2-(difluoromethyl)-4-(2-methylpropyl)-6-(trifluoromethyl)-3,5-pyridinedicarbothioate(MON 15151);N,N-diethyl-2-(Inaphthalenyloxy)propanamide (napropamide); 4-(dipropylamino)-3,5-dinitro-benzenesulfonamide (oryzalin); 3-[2,4-dichloro-5-(1-methylethoxy)phenyl]-5-(1,1-dimethylethyl)-1,3,4-oxadiazol-2-(3H)-one (oxadiazon); 2-chloro-1-(3-ethoxy-4-nitrophenoxy)-4(trifluoromethyl) benzene (oxyfluorfen); N-(1-ethylpropyl)-3,4-dimethyl-2,6-dinitrobenzenamine (pendimethalin); $\mathrm{N}^{3}, \mathrm{~N}^{3}$-di-n-propyl-2,4-dinitro-6-(trifluoromethyl)-m-phenylenediamine (prodiamine); 2,6-dinitro-N,N-dipropyl-4-(trifluoromethyl)benzenamine (trifluralin).

Landscaping with ornamental grasses, such as pampas grass, tufted hair grass, blue fescue, fountain grass, and ribbon grass, has increased in popularity in recent years. To economically produce plants in sufficient numbers to supply this growing demand, nursery workers need a safe and effective

Received for publication 13 Dec. 1989. We thank Colleen M. Kearns for her excellent technical assistance. We also thank Peat \& Sons Nursery, Huntington, N. Y., and Richard Simon, Blue Mount Nurseries, Monkton, Md., for donating plant materials used in this study. We also thank Richard Biamonte and W.R. Grace \& Co. for donating potting mix. Research was supported by grants from the Long Island Nurserymen's Association, Eli Lilly \& Co., Monsanto Agricultural Co., O.M. Scott \& Sons, and Rhone-Poulenc Ag Co.; and through Hatch Project no. 141434. The cost of publishing this paper was defrayed in part by the payment of page charges. Under postal regulations, this paper therefore must be hereby marked advertisement solely to indicate this fact. 'Assistant Professor of Weed Science. ${ }^{2}$ Extension Weed Control Specialist.

control program. Hand weeding cost 12 times as much as an effective her- bicide program (Neal and Skroch, 1984); however, little is known of the herbicide tolerance of ornamental grasses. Similarly, no reports are published concerning herbicide phytotoxicity to beach grass, a popular dune stabilization species.

Of these ornamental grasses, more is known of pampas grass tolerance to herbicides. Glaze et al. $(1980,1981)$ observed that alachlor did not affect pampas grass growth when applied at $<9 \mathrm{~kg}$ a.i./ha. Similarly, Glaze and Singh (1989) observed no injury to pampas grass from a single application of metolachlor up to $17.9 \mathrm{~kg}$ a.i./ha, although repeated applications at lower rates, 2.2 to $9.0 \mathrm{~kg}$ a.i./ha, reduced pampas grass growth and quality. Metolachlor (Pennant herbicide, Ciba-Geigy, Greensboro, N.C.) is currently labeled for use on pampas grass in field production nurseries and landscapes, but not in containers. The granular combination of oxyfluorfen plus pendimethalin (Scotts Ornamental Herbicide II, O.M. Scott \& Sons, Marysville, Ohio) is labeled for use in container- and field-grown pampas grass. However, pampas grass was injured by oxyfluorfen when applied as a spray or injected into the irrigation (Glaze et al., 1980). Oryzalin also. injured pampas grass (Glaze et al., 1981). A recent literature search revealed no other reports of preemergent herbicide safety on ornamental grasses. Possibly herbicides, such as pendimethalin (Herrick and Ilnicki, 1985), benefin (Jagschitz, 1985), benefin plus trifluralin (Sawyer and Jagschitz, 1987), isoxaben (Colbert and Ford, 1987), MON 15100 (Parrish et al., 1988), prodiamine (Hummel et al., 1990), and oxadiazon (Sawyer and Jagschitz, 1987), that are safe on turfgrasses might also be safe on ornamental landscape grasses. However, these products have not been tested for safety on ornamental landscape grasses.

The objective of this study was to investigate the safety of preemergent herbicides on commonly grown ornamental grasses, with emphasis on herbicides that are currently labeled or under development for nursery or turf uses.

"Two experiments were conducted, one in

Table 1. Herbicides, formulations, and rates used in 1988 and 1989 tests

\begin{tabular}{|c|c|c|}
\hline Herbicide & Formulation & $\begin{array}{c}\text { Rate } \\
(\mathrm{kg} \mathrm{a.i./ha)}\end{array}$ \\
\hline Benefin + trifluralin & $\begin{array}{l}2 \mathrm{G}(1.33 \% \text { benefin }+0.67 \% \text { trifluralin combination } \\
\text { granule) }\end{array}$ & 2.2 \\
\hline Isoxaben & $75 \mathrm{DF}$ ( $75 \%$ dry flowable) & $0.56,1.1$ \\
\hline Isoxaben + oryzalin & $80 \mathrm{DF}$ ( $20 \%$ isoxaben $+60 \%$ oryzalin dry flowable) & $3.4,4.5$ \\
\hline Isoxaben + trifluralin & $\begin{array}{l}2.5 \mathrm{G}(0.5 \% \text { isoxaben }+2 \% \text { trifluralin combination }) \\
\text { granule) }\end{array}$ & 4.2 \\
\hline Metolachlor & $8 \mathrm{EC}\left(0.96 \mathrm{~kg} \cdot\right.$ liter $^{-1}$ emulsifiable concentrate $)$ & $3.4,6.7$ \\
\hline Metolachlor & $5 \mathrm{G}$ ( $5 \%$ granule $)$ & 6.7 \\
\hline MON 15151 & $1 \mathrm{EC}\left(0.12 \mathrm{~kg} \cdot\right.$ liter $^{-1}$ emulsifiable concentrate $)$ & $0.56,1.1,2.2^{z}$ \\
\hline Napropamide & $50 \mathrm{WP}(50 \%$ wettable powder $)$ & $2.2,4.5$ \\
\hline Napropamide & $5 \mathrm{G}(5 \%$ granule $)$ & $4.5^{2}$ \\
\hline Oryzalin & 4AS $\left(0.48 \mathrm{~kg} \cdot \operatorname{liter}^{-1}\right.$ aqueous suspension) & $2.2,4.5$ \\
\hline Oxadiazon & $2 \mathrm{G}(2 \%$ granule $)$ & $2.2,4.5$ \\
\hline Oxadiazon & $50 W P(50 \%$ wettable powder) & 2.2 \\
\hline Oxyfluorfen i pendimethalin & $\begin{array}{l}3 \mathrm{G} \text { ( } 2 \% \text { oxyfluorfen }+1 \% \text { pendimethalin combination } \\
\text { granule) }\end{array}$ & 3.4 \\
\hline Pendimethalin & $60 \mathrm{DG}$ (60\% water-dispersible granule) & $2.2,4.5$ \\
\hline Prodiamine & 65DG (65\% water-dispersible granule) & $1.1,2.2$ \\
\hline Trifluralin & $5 \mathrm{G}(5 \%$ granule $)$ & $2.2,4.5$ \\
\hline
\end{tabular}

${ }^{2}$ Napropamide $5 \mathrm{G}$ at $4.5 \mathrm{~kg}$ a.i./ha and MON 15151 at $2.2 \mathrm{~kg}$ a.i./ha were included in 1989 only. 
Table 2. Preemergent herbicide phytotoxicity to container-grown ornamental grasses (1988).

\begin{tabular}{|c|c|c|c|c|c|c|c|c|}
\hline \multirow[b]{2}{*}{ Herbicide $^{y}$} & \multirow[b]{2}{*}{ Formulation } & \multirow[b]{2}{*}{$\begin{array}{c}\text { Rate } \\
\text { (kg a.i. } / \mathrm{ha}) \\
\end{array}$} & \multicolumn{6}{|c|}{ Plant quality ${ }^{z}$} \\
\hline & & & $\begin{array}{c}\text { Tufted } \\
\text { hair grass }\end{array}$ & $\begin{array}{c}\text { Ribbon } \\
\text { grass }\end{array}$ & $\begin{array}{c}\text { Beach } \\
\text { grass }\end{array}$ & $\begin{array}{c}\text { Blue } \\
\text { fescue }\end{array}$ & $\begin{array}{c}\text { Fountain } \\
\text { grass }\end{array}$ & $\begin{array}{c}\text { Pampas } \\
\text { grass }\end{array}$ \\
\hline None & -.. & -.- & 100 & 100 & 100 & 100 & 100 & 100 \\
\hline \multirow[t]{2}{*}{ Isoxaben } & $75 \mathrm{DF}$ & 0.56 & 100 & 100 & 93 & 100 & 100 & 100 \\
\hline & 75DF & 1.1 & 100 & 100 & $98^{\circ}$ & 100 & 98 & 95 \\
\hline \multirow{2}{*}{$\begin{array}{c}\text { Isoxaben }+ \\
\text { oryzalin }\end{array}$} & & & & & & & & \\
\hline & $\begin{array}{l}\text { 80DF } \\
\text { 80DF }\end{array}$ & $\begin{array}{l}3.3 \\
4.5\end{array}$ & $\begin{array}{l}73 \\
68\end{array}$ & $\begin{array}{l}93 \\
88\end{array}$ & $\begin{array}{r}98 \\
100\end{array}$ & $\begin{array}{l}13 \\
23\end{array}$ & $\begin{array}{l}83 \\
68\end{array}$ & $\begin{array}{l}68 \\
40\end{array}$ \\
\hline $\begin{array}{l}\text { Isoxaben }+ \\
\text { trifluralin }\end{array}$ & $2.5 \mathrm{G}$ & 4.2 & 93 & 100 & 93 & 95 & 100 & 98 \\
\hline \multirow[t]{2}{*}{ Metolachlor } & $8 \mathrm{EC}$ & 3.4 & 80 & 78 & 95 & 80 & 58 & 100 \\
\hline & $\begin{array}{c}8 \mathrm{EC} \\
5 \mathrm{G}\end{array}$ & $\begin{array}{l}6.7 \\
6.7\end{array}$ & $\begin{array}{l}60 \\
68\end{array}$ & $\begin{array}{l}43 \\
55\end{array}$ & $\begin{array}{l}78 \\
98\end{array}$ & $\begin{array}{l}45 \\
20\end{array}$ & $\begin{array}{l}43 \\
48\end{array}$ & $\begin{array}{l}78 \\
83\end{array}$ \\
\hline \multirow[t]{2}{*}{ MON 15151} & 1EC & 0.56 & 98 & 100 & 98 & 90 & 100 & 100 \\
\hline & $1 \mathrm{EC}$ & 1.1 & 88 & 100 & 100 & 73 & 98 & 100 \\
\hline \multirow{2}{*}{ Napropamide } & 50WP & 2.2 & 88 & 95 & 98 & 95 & 100 & 100 \\
\hline & $50 \mathrm{WP}$ & 4.5 & 75 & 90 & 88 & 80 & 93 & 93 \\
\hline \multirow[t]{2}{*}{ Oryzalin } & $4 \mathrm{AS}$ & 2.2 & 80 & 85 & 83 & 23 & 80 & 60 \\
\hline & 4AS & 4.5 & 55 & 75 & 85 & 0 & 75 & 38 \\
\hline \multirow[t]{3}{*}{ Oxadiazon } & $2 \mathrm{G}$ & 2.2 & 100 & 100 & 88 & 100 & 100 & 100 \\
\hline & $2 \mathrm{G}$ & 4.5 & 100 & 100 & 88 & 98 & 90 & 85 \\
\hline & $50 \mathrm{WP}$ & 2.2 & 100 & 100 & 100 & 100 & 100 & 100 \\
\hline \multirow[t]{2}{*}{ Trifluralin } & $5 \mathrm{G}$ & 2.2 & 93 & 100 & 98 & 100 & 95 & 100 \\
\hline & $5 G$ & 4.5 & 95 & 83 & 100 & 90 & 100 & 100 \\
\hline \multicolumn{3}{|c|}{ LSD $(K$ ratio $=100)$} & 15 & 13 & 17 & 22 & 18 & 16 \\
\hline
\end{tabular}

${ }^{2}$ Plant quality was visually evaluated 15 weeks after treatment using a percent scale, where $\mathrm{O}=$ dead plants and $100=$ the best growth under the test conditions. Data represent means of four, three-plant replicates.

${ }^{y}$ Other herbicides that caused no injury: prodiamine, pendimethalin, benefin + trifluralin, and oxyfluorfen + pendimethalin.

Table 3. Preemergent herbicide phytotoxicity to container-grown ornamental grasses (1989).

\begin{tabular}{|c|c|c|c|c|c|c|c|c|}
\hline \multirow[b]{2}{*}{ Herbicide $y$} & \multirow[b]{2}{*}{ Formulation } & \multirow[b]{2}{*}{$\begin{array}{c}\text { Rate } \\
\text { (kg a.i.//ha) }\end{array}$} & \multicolumn{6}{|c|}{ Plant quality ${ }^{z}$} \\
\hline & & & $\begin{array}{c}\text { Tufted } \\
\text { hair grass }\end{array}$ & $\begin{array}{l}\text { Ribbon } \\
\text { grass }\end{array}$ & $\begin{array}{l}\text { Beach } \\
\text { grass }\end{array}$ & $\begin{array}{c}\text { Blue } \\
\text { fescue }\end{array}$ & $\begin{array}{c}\text { Fountain } \\
\text { grass }\end{array}$ & $\begin{array}{c}\text { Pampas } \\
\text { grass }\end{array}$ \\
\hline$\overline{\text { None }}$ & --- & $\ldots$ & 90 & 98 & 85 & 100 & 100 & 98 \\
\hline \multirow[t]{2}{*}{ Isoxaben } & $75 \mathrm{DF}$ & 0.56 & 95 & 98 & 98 & 85 & 98 & 98 \\
\hline & $75 \mathrm{DF}$ & 1.1 & 90 & 95 & 92 & 92 & 98 & 95 \\
\hline \multirow{2}{*}{$\begin{array}{c}\text { Isoxaben }+ \\
\text { oryzalin }\end{array}$} & & & & & & & & \\
\hline & $\begin{array}{l}\text { 80DF } \\
\text { 80DF }\end{array}$ & $\begin{array}{l}3.4 \\
4.5\end{array}$ & $\begin{array}{l}72 \\
68\end{array}$ & $\begin{array}{l}82 \\
85\end{array}$ & $\begin{array}{l}92 \\
85\end{array}$ & $\begin{array}{l}20 \\
40\end{array}$ & $\begin{array}{l}12 \\
20\end{array}$ & $\begin{array}{l}38 \\
30\end{array}$ \\
\hline \multirow{2}{*}{$\begin{array}{l}\text { Isoxaben }+ \\
\text { trifluralin }\end{array}$} & & & & & & & & \\
\hline & $2.5 \mathrm{G}$ & 4.2 & 90 & 90 & 95 & 78 & 88 & 90 \\
\hline \multirow[t]{3}{*}{ Metolachlor } & $8 \mathrm{EC}$ & 3.4 & 68 & 70 & 90 & 42 & 62 & 72 \\
\hline & $8 \mathrm{EC}$ & 6.7 & 62 & 45 & 75 & 10 & 38 & 68 \\
\hline & $5 \mathrm{G}$ & 6.7 & 58 & 58 & 88 & 10 & 45 & 72 \\
\hline \multirow{3}{*}{ MON 15151} & $1 \mathrm{EC}$ & 0.56 & 95 & 90 & 95 & 82 & 100 & 95 \\
\hline & $1 \mathrm{EC}$ & 1.1 & 82 & 98 & 88 & 85 & 98 & 98 \\
\hline & $1 \mathrm{EC}$ & 2.2 & 62 & 82 & 85 & 40 & 10 & 60 \\
\hline \multirow[t]{3}{*}{ Napropamide } & $50 W P$ & 2.2 & 92 & 98 & 100 & 70 & 88 & 92 \\
\hline & $50 \mathrm{WP}$ & 4.5 & 72 & 82 & 100 & 60 & 68 & 72 \\
\hline & $5 \mathrm{G}$ & 4.5 & 68 & 85 & 98 & 75 & 92 & 92 \\
\hline \multirow[t]{3}{*}{ Oxadiazon } & $2 \mathrm{G}$ & 2.2 & 100 & 95 & 95 & 92 & 100 & 98 \\
\hline & $2 \mathrm{G}$ & 4.5 & 100 & 100 & 100 & 78 & 88 & 92 \\
\hline & $50 \mathrm{WP}$ & 2.2 & 100 & 98 & 100 & 85 & 90 & 92 \\
\hline \multirow[t]{2}{*}{ Oryzalin } & $4 \mathrm{AS}$ & 2.2 & 85 & 100 & 90 & 45 & 45 & 45 \\
\hline & $4 \mathrm{AS}$ & 4.5 & 70 & 78 & 78 & 35 & 12 & 20 \\
\hline \multirow[t]{2}{*}{ Trifluralin } & $5 G$ & 2.2 & 88 & 95 & 90 & 82 & 98 & 95 \\
\hline & $5 \mathrm{G}$ & 4.5 & 98 & 95 & 95 & 80 & 85 & 92 \\
\hline \multicolumn{2}{|c|}{$\operatorname{LSD}(K$ ratio $=100)$} & & 15 & 12 & 14 & 23 & 15 & 13 \\
\hline
\end{tabular}

${ }^{2}$ Plant quality was visually evaluated 12 weeks after treatment using a percent scale, where $\mathrm{O}=$ dead plants and $100=$ the best growth under the test conditions. Data represent means of four, three-plant replicates.

'Other herbicides that caused no injury: prodiamine, pendimethalin, benefin + trifluralin, and oxyfluorfen + pendimethalin.

1988 and the other in 1989, at the Cornell Univ. Ornamental Test Garden, Ithaca, N. Y., to investigate herbicide safety on six species of ornamental grasses. Beach grass, tufted hair grass, and ribbon grass were propagated by division. Blue fescue, foun- $(\mathrm{v} / \mathrm{v})]$. Seedlings were grown in a greenhouse maintained between 18 and 24C. A 16-h photoperiod was maintained with high-pressure sodium lights supplying $\approx 350$ $\mu \mathrm{mol} \cdot \mathrm{s}^{-1} \cdot \mathrm{m}^{-2}$ photosynthetically active radiation at the plant level. Plants were well established before removal from the greenhouse for potting and treatment.

Plant propagules, divisions and greenhouse-grown liners, were potted in 2.8-liter plastic pots using a commercially available soilless medium (Metro 500, W.R. Grace \& Co., Fogelsville, Pa.). Potting was on 15 June 1988 and 17 May 1989. Herbicides were applied within $48 \mathrm{~h}$ of potting. Plants were irrigated after potting and twice daily thereafter with an automated overhead irrigation system. Plants were fertilized twice during the growing season with $\approx 8 \mathrm{~g} / \mathrm{pot}$ of a $20 \mathrm{~N}$ 3.6P-10K sulfur-coated fertilizer (Lesco 206-12 Fertilizer plus Minors, Lesco, Rocky River, Ohio). Herbicide sprays were applied with a $\mathrm{CO}_{2}$-pressurized backpack sprayer equipped with flat fan nozzles and calibrated to deliver 280 liters -ha-' at $276 \mathrm{kPa}$. Granular herbicides were applied in preweighed aliquots using a hand-held shaker jar. Herbicide formulations and rates of application are listed in Table 1; all quantities are given as the active ingredient. Herbicide treatments were applied in a randomized complete block design with four replicates. Each experimental unit contained three pots of each species.

Plant quality was visually evaluated twice, $\approx 6$ weeks after treatment and before fresh weight harvest, on a percent vigor scale, where $0=$ dead plants, $50=50 \%$ growth reduction and injury, and $100=$ the best growth under the test conditions. Above-ground fresh weights were measured 15 weeks after treatment in 1988 and 12 weeks after treatment in 1989. Data were subjected to analysis of variance and means were separated using the Wailer/Duncan procedure (SAS, 1985). Species were analyzed separately.

The results between years were similar. As visual ratings and fresh weight data were highly correlated $(P=0.0001)$ and coefficients of variability for the visual ratings were generally lower than for fresh weight data, only the final visual ratings, 15 weeks after treatment in 1988 and 12 weeks after treatment in 1989, are presented.

The highest rate of oryzalin applied alone injured all species in both years and the lower rate frequently was injurious (Tables 2 and 3). Plant injury was greater at 4.5 than at $2.2 \mathrm{~kg}$ a.i./ha (Table 3). Glaze et al. (1981) observed a similar rate response in oryzalin phytotoxicity to pampas grass. Injury also occurred when oryzalin was combined with isoxaben, except for beach grass. Ribbon grass was not injured by the combination in 1988, but was injured in 1989 (Tables 2 and 3). Isoxaben applied alone or combined with trifluralin caused no injury in either year; therefore, phytotoxicity was attributed to oryzalin.

In 1988, metolachlor 8EC and 5G at 6.7 $\mathrm{kg} \cdot \mathrm{ha}^{-1}$ injured all species except beach grass, 
which was tolerant of the $5 \mathrm{G}$ formulation (Table 2). Beach grass, fescue, and pampas grass were not injured at $3.4 \mathrm{~kg} \cdot \mathrm{ha}^{-1}$. In 1989 , all species were injured by metolachlor, regardless of rate or formulation, except beach grass, which tolerated the $5 \mathrm{G}$ formulation and the $3.4-\mathrm{kg} \cdot \mathrm{ha}^{-1}$ rate of the $8 \mathrm{EC}$ formulation (Table 3 ). The symptoms of metolachlor injury included crinkled leaves and inhibited unfurling of the new leaves, similar to symptoms described for acetanilide herbicide injury on corn (Jennings and Nyvall, 1983). As metolachlor is labeled for field-grown pampas grass, it is particularly noteworthy that pampas grass was injured by metolachlor in this study. It is likely that established, field-grown plants would be less sensitive to herbicide injury than would be the tender, greenhouse-grown plants used in this study.

Napropamide 50WP at $4.5 \mathrm{~kg} \cdot \mathrm{ha}^{-1}$ caused some phytotoxicity to beach grass, tufted hair grass, and blue fescue in 1988. At the 6week rating (data not shown), the quality of these species was reduced, but beach grass and blue fescue recovered by 15 weeks (Table 2). In 1989, napropamide 50WP at 4.5 $\mathrm{kg} \cdot \mathrm{ha}^{-1}$ injured all species except beach grass (Table 3). Only blue fescue was injured at $2.2 \mathrm{~kg} \cdot \mathrm{ha}^{-1}$. The $5 \mathrm{G}$ formulation was safe on beach grass, fountain grass, and pampas grass, but injured tufted hair grass, blue fescue, and ribbon grass.

In 1988, blue fescue was slightly injured by MON 15151 at $1.1 \mathrm{~kg} \cdot \mathrm{ha}^{-1}$ but not by $0.56 \mathrm{~kg} \cdot \mathrm{ha}^{-1}$ (Table 2). The other species were not injured. In 1989, the $1.1-\mathrm{kg} \cdot \mathrm{ha}^{-1}$ rate was safe on all species, but $2.2 \mathrm{~kg} \cdot \mathrm{ha}^{-1}$ injured all species (Table 3 ).

Oxadiazon at $4.5 \mathrm{~kg}$-ha-1 caused some temporary injury to fescue, $20 \%$ injury 6 weeks after treatment in 1988 (data not shown), but plants had recovered by 15 weeks (Table 2). No oxadiazon injury was observed in 1989.

Herbicides that caused no significant injury to these ornamental grasses in either year were: isoxaben, pendimethalin, prodiamine, benefin plus trifluralin, isoxaben plus trifluralin, and oxyfluorfen plus pendimethalin (data not shown). Trifluralin was safe on all species at $2.2 \mathrm{~kg} \cdot \mathrm{ha}^{-1}$, but the $4.5-\mathrm{kg} \cdot \mathrm{ha}^{-1}$ rate reduced ribbon grass quality by $17 \%$ in 1988 and fountain grass quality by $15 \%$ in 1989 (Tables 2 and 3). The other species were not injured by trifluralin at $4.5 \mathrm{~kg} \cdot \mathrm{ha}^{-1}$.

We have shown that the ornamental grasses tested have considerable tolerance to many nursery and turf herbicides. Notable exceptions were oryzalin, metolachlor, and napropamide. Beach grass, blue fescue, fountain grass, pampas grass, and ribbon grass tolerated recommended use-rates of trifluralin, pendimethalin, prodiamine, oxadiazon, isoxaben, benefin plus trifluralin, and oxyfluorfen plus pendimethalin. Some variability in plant vigor and growth between years was observed for trifluralin and oxadiazon treatments. Reasons for this variability are unknown. Before labeling, further research should be conducted under a variety of conditions to confirm herbicide safety. Also, ad- ditional testing is needed on other species of ornamental grasses.

\section{Literature Cited}

Colbert, F.O. and D.H. Ford. 1987. Isoxaben for broadleaf weed control in ornamentals, turf, and nonbearing trees and vines. Proc. Western Weed Sci. Soc. 40:155-163.

Glaze, N.C. and M. Singh. 1989. Ornamental response to two methods of metolachlor application. Proc. Weed Sci. Soc. Amer. 29:32.

Glaze, N.C., M. Singh, and S.C. Phatak. 1980 Response of pampas grass and two azalea cultivars to alachlor, oxadiazon and oxyfluorfen. Proc. Weed Sci. Soc. Amer. 20:41-42.

Glaze, N.C., M. Singh, and S.C. Phatak. 1981. Oryzalin for weed control in container-grown pittosporum, cleyera, gardinia, pampas grass, liriope, and scuba. Proc. Southern Nurserymens Assn. Res. Conf. 26:235.

Herrick, R.M. and R.D. Ilnicki. 1985. Preemergent control of crabgrass in turf with pendimethalin. Proc. Northeastern Weed Sci. Soc. 39:271-272.
Hummel, N.W., Jr., M.C. Fowler, and J.C. Neal. 1990. Prodiamine effects on quality and rooting of Kentucky bluegrass turf. Crop Sci. 30:976979.

Jagschitz, J.A. 1985. Crabgrass control in turfgrass with herbicides. Proc. Northeastern Weed Sci. Soc. 39:274-278.

Jennings, V.M. and R.F. Nyvall. 1983. Misapplied row crop herbicides, symptoms and problems. Iowa State Univ. Coop. Ext. Ser. Pm738.

Neal, J.C. and W.A. Skroch. 1984. Comparative weed control for container-grown compacta holly. Proc. Southern Nurserymen Assn. Res. Conf. 29:240-243.

Parrish, S.K., R.J. Thompson, D.R. Williamson, and S.J. Stehling. 1988. MON 15100-A new herbicide for turf. Proc. Weed Sci. Soc. Amer. 28:32.

SAS Institute, Inc. 1985. SAS user's guide: Statistics, version 5 ed. SAS Institute, Inc., Cary, N.C.

Sawyer, C.D. and J.A. Jagschitz. 1987. Timing of preemergence herbicides for crabgrass control. Proc. Northeastern Weed Sci. Soc. 41:211212. 